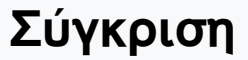

Tóp. 24 (2014)

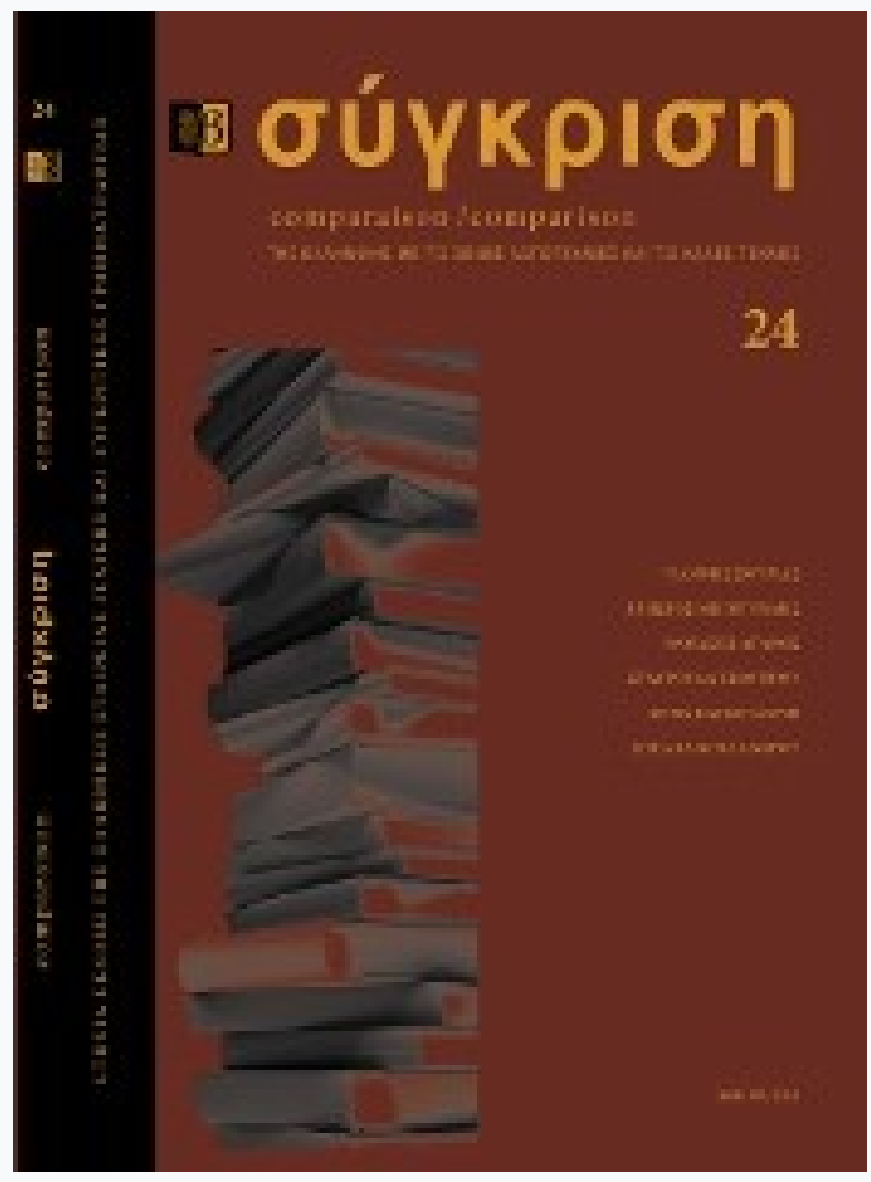

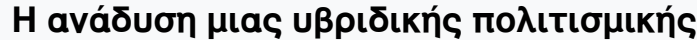

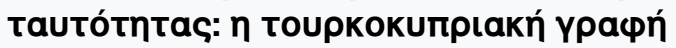

\section{Avva Katoíylavvn}

doi: $10.12681 /$ comparison.17

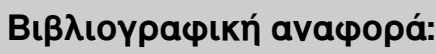

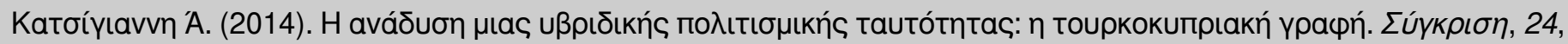
75-81. https://doi.org/10.12681/comparison.17 


\section{ANNAKATEITIANNH}

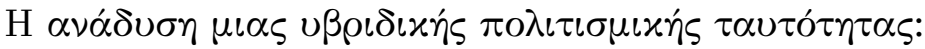 \\ $\eta \tau о \nu \rho x о х v \pi \rho \iota \alpha x \dot{~} \gamma \rho \alpha \varphi \dot{n}$}

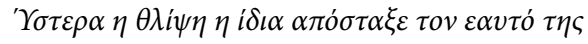

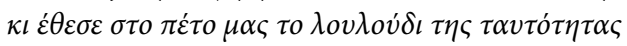

$\Phi \iota \lambda i \zeta N a \lambda v \tau \tau \beta \beta \dot{\varepsilon} v$

$\mathrm{H}$

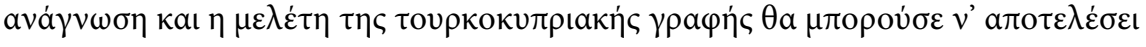

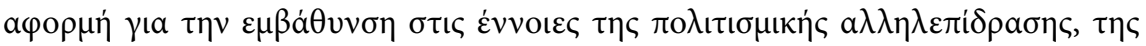

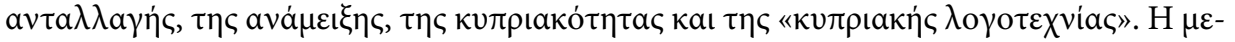

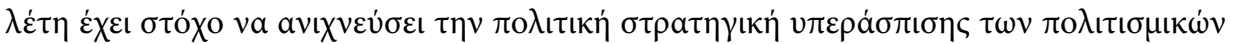

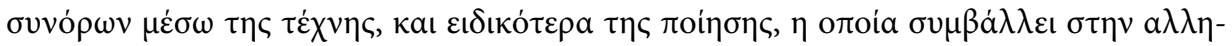

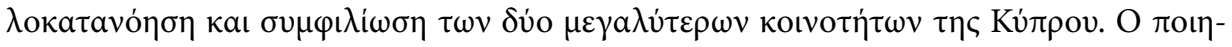

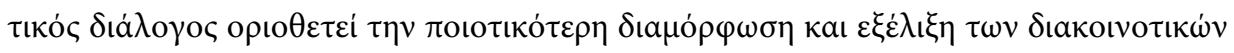

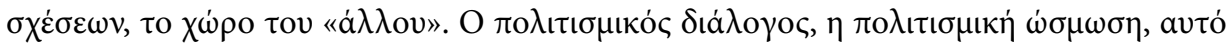

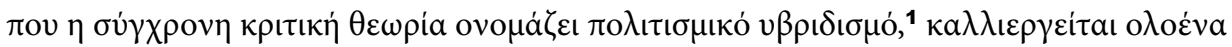

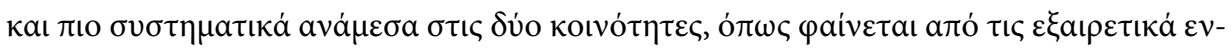

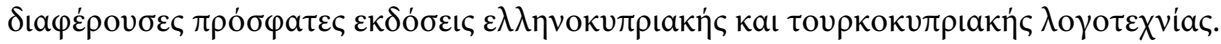

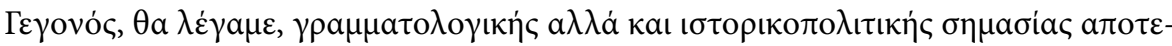

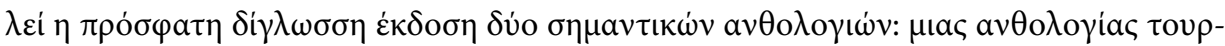

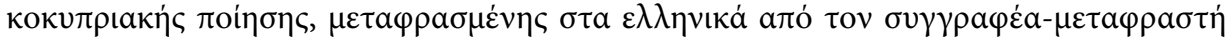

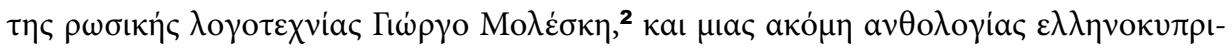

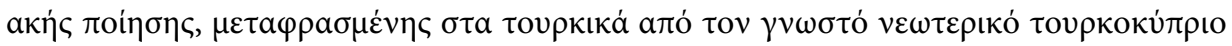

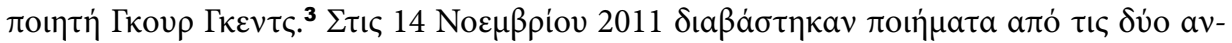

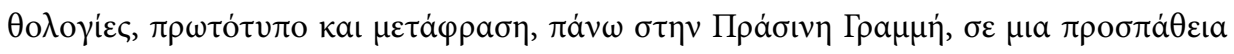

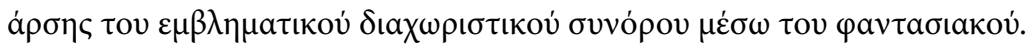

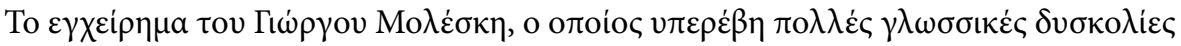
үıа va a

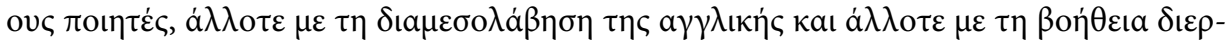

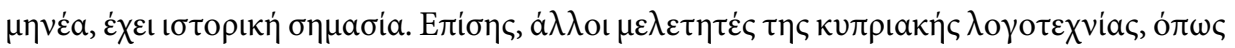

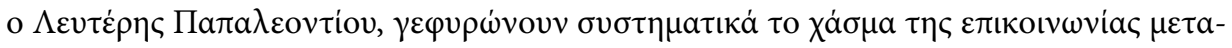

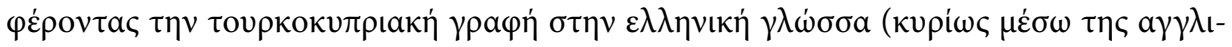

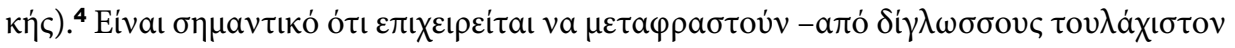




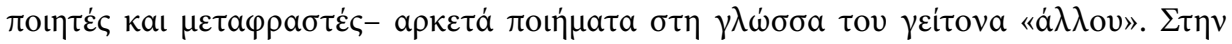

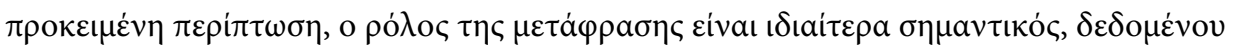

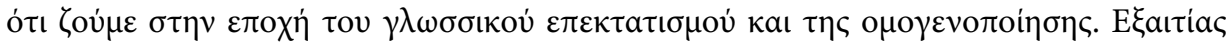

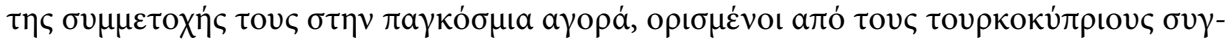

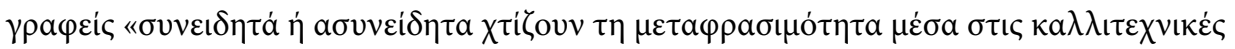

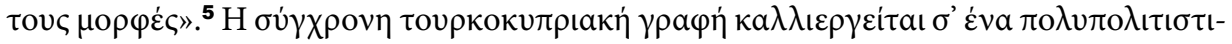

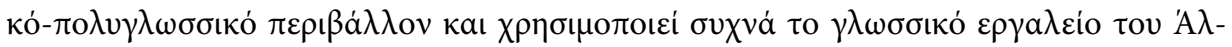

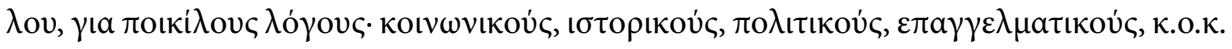

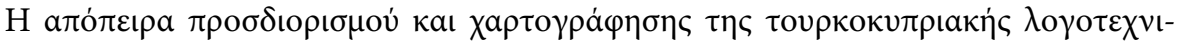

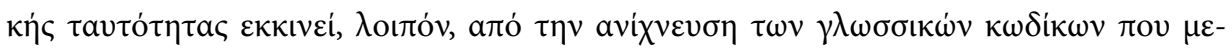

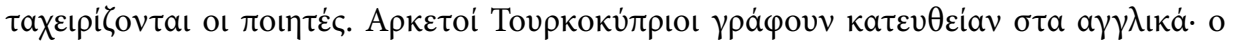

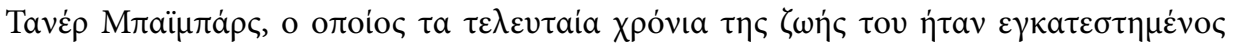

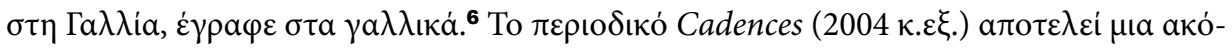

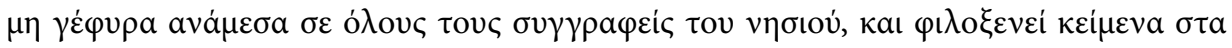

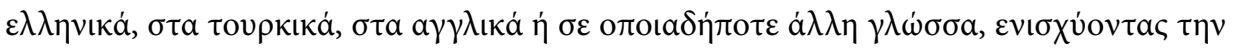
$\pi \mathrm{o} \lambda v \gamma \lambda \omega \sigma \sigma i \alpha$.

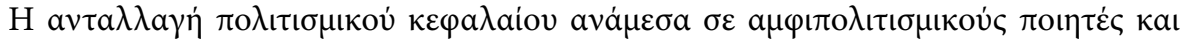

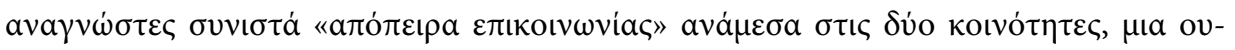

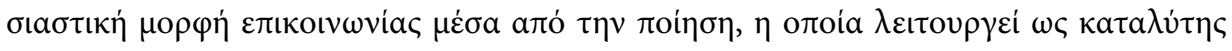

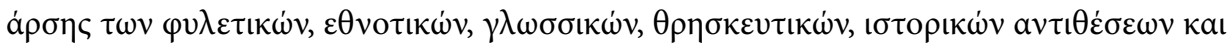

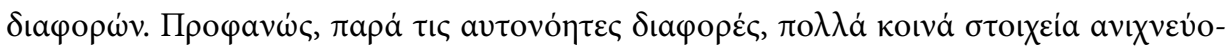

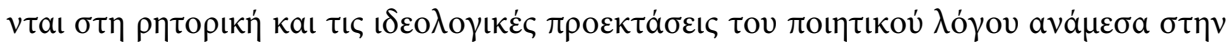

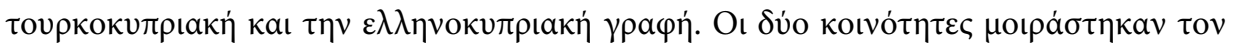

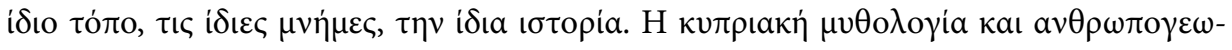

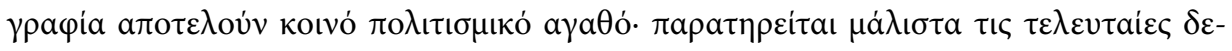

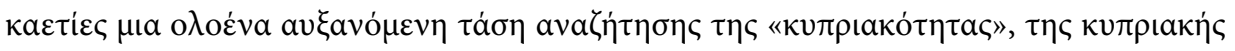

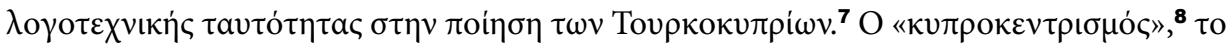

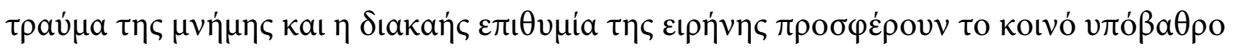
$\tau \eta \varsigma \pi \mathrm{o} \lambda \iota \tau \iota \sigma \mu \iota \kappa \dot{\varsigma} \varsigma \omega \dot{\sigma} \mu \omega \sigma \eta \varsigma$.

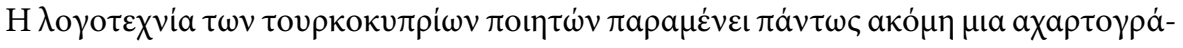

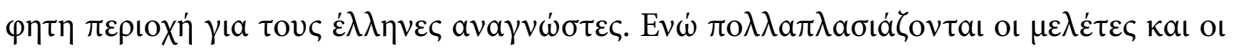

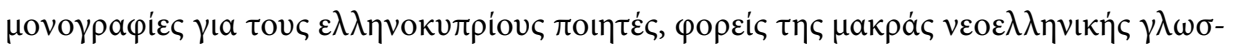

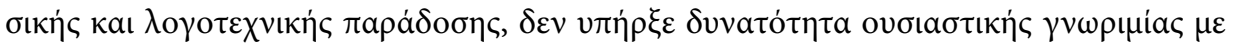

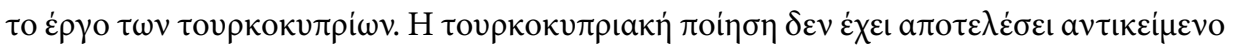

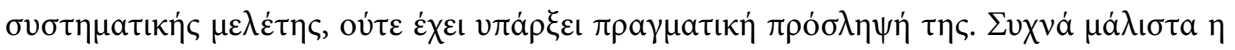

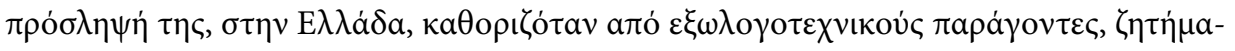

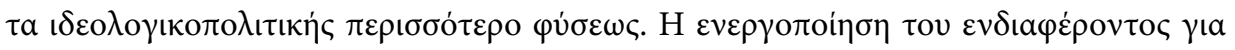




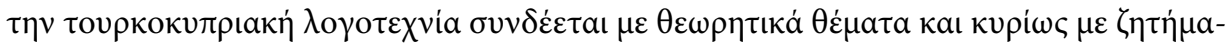

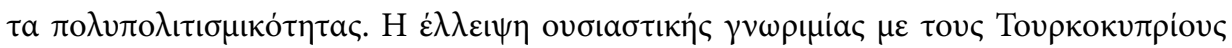

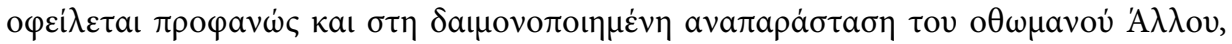

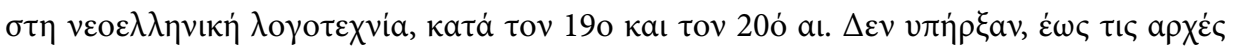

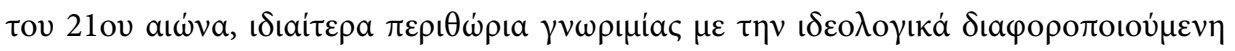

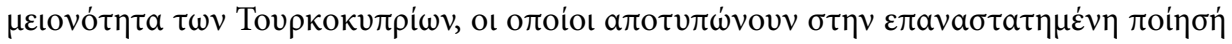

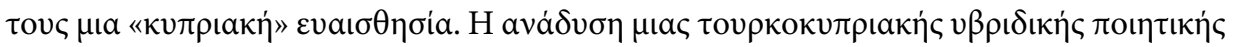

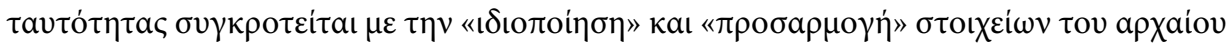

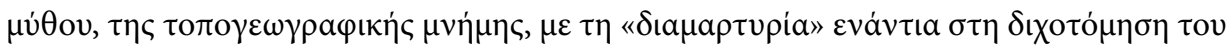

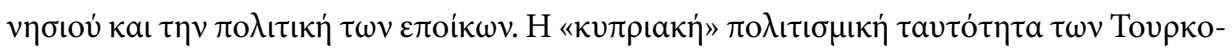

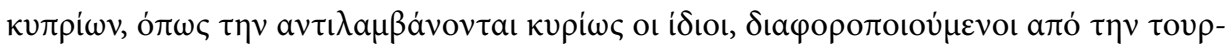

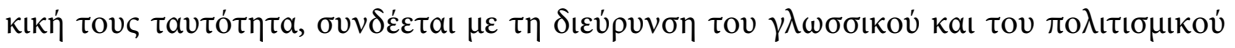

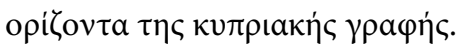

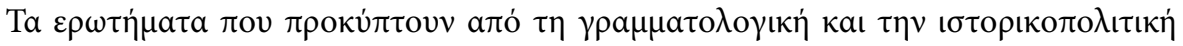

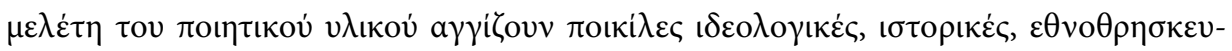

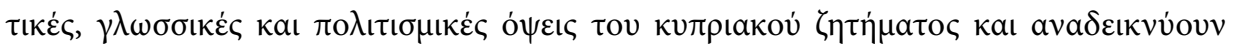

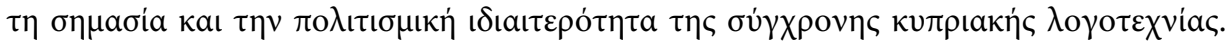

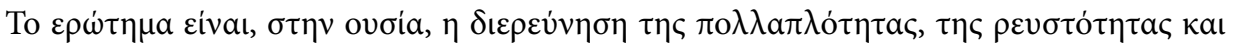

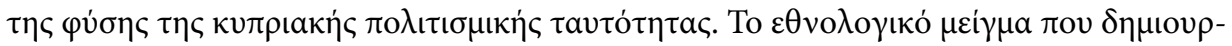

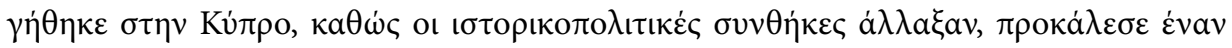

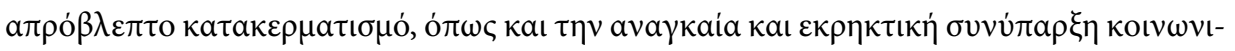

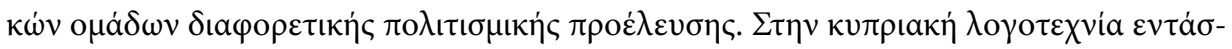

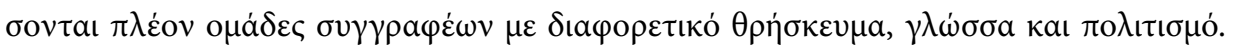

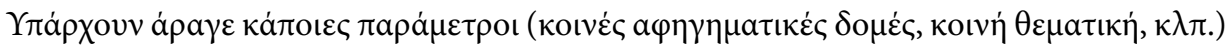

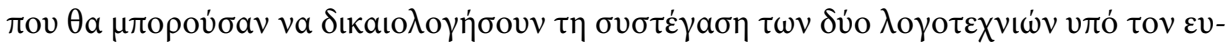

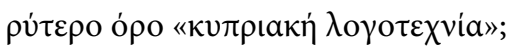

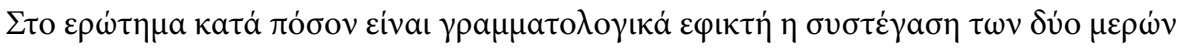

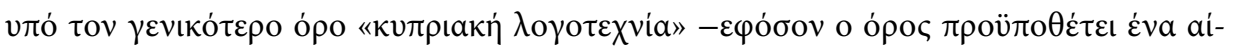

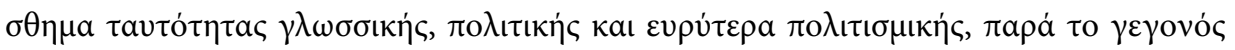

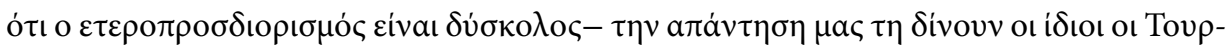

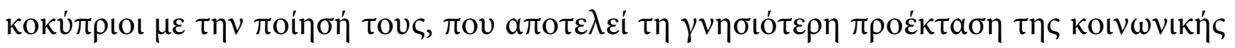

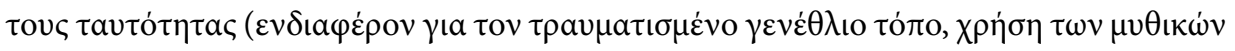

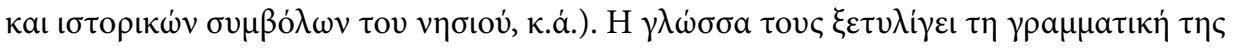

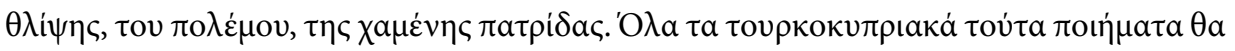
$\mu \pi$ o

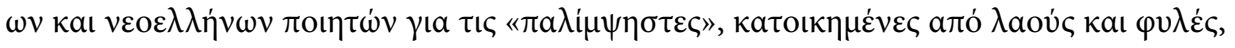

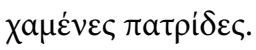




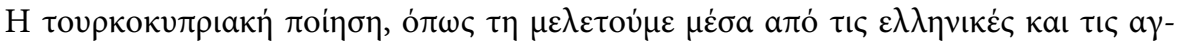

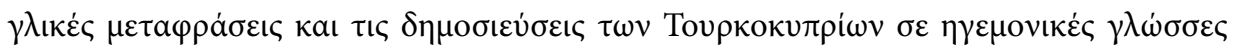

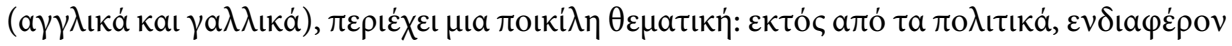

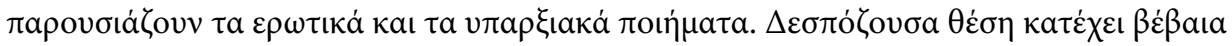
$\eta \theta \varepsilon \mu \alpha \tau$ ๆ

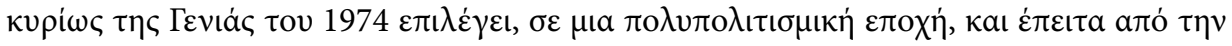

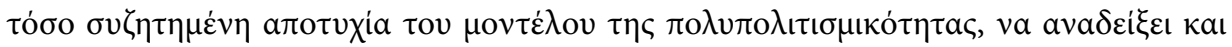

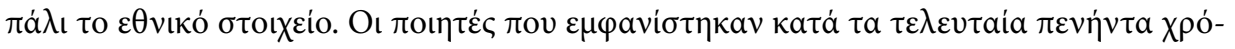

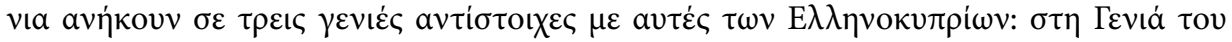

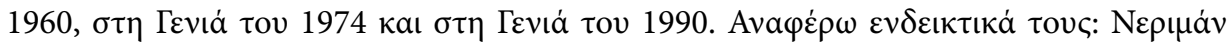

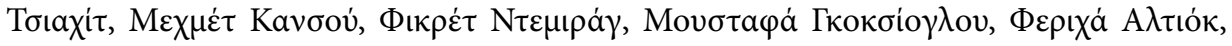

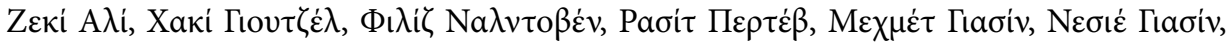

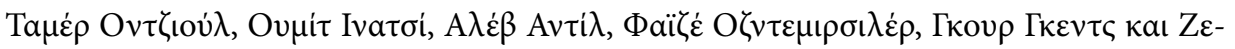
váv $\sum \varepsilon \lambda \tau \sigma ı$ เón $^{9}$

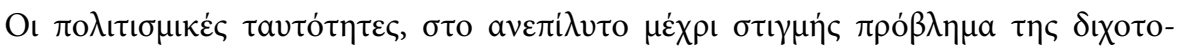

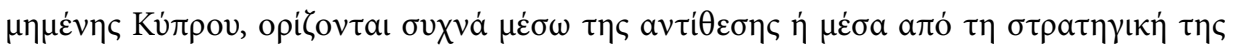

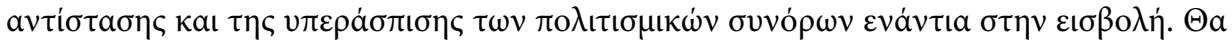

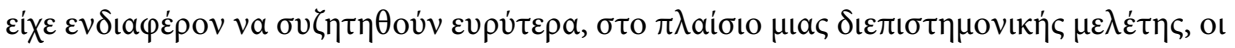

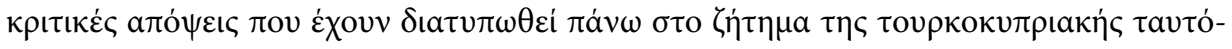

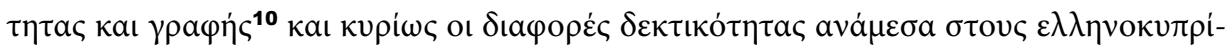

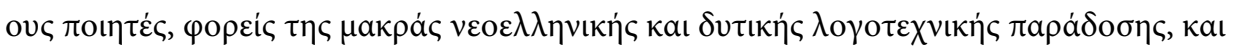

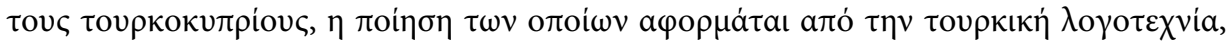

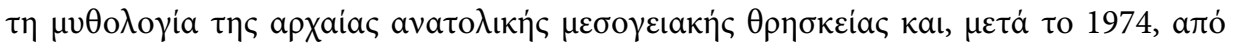

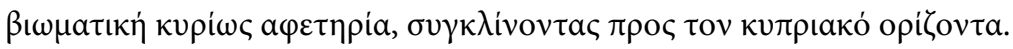

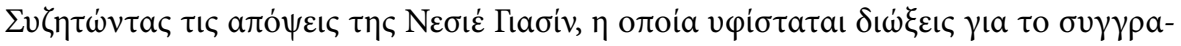

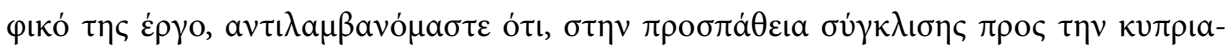

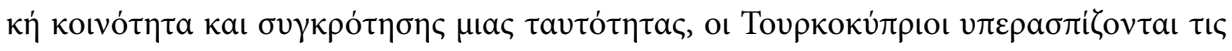

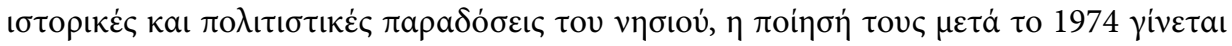

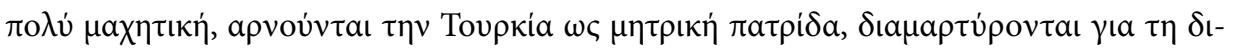

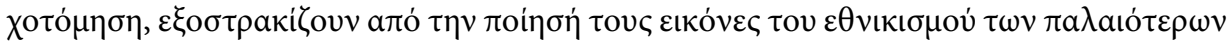

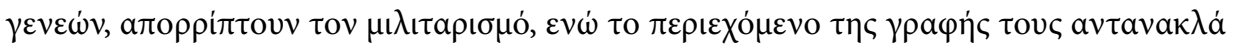

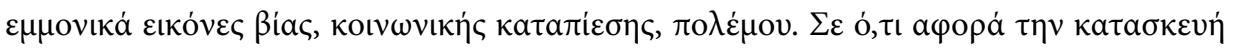

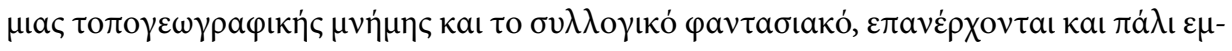

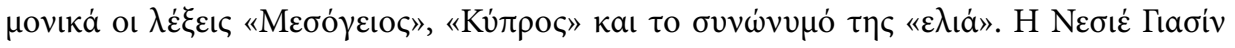

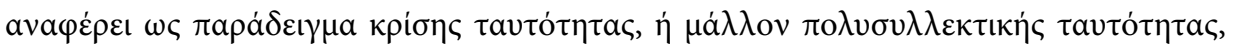

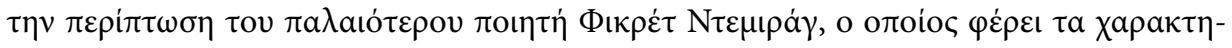

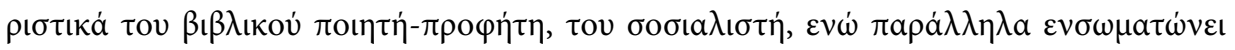




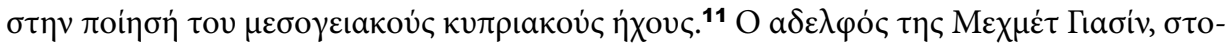

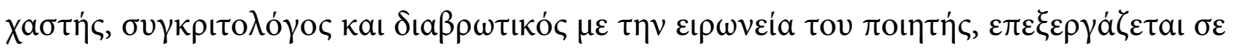

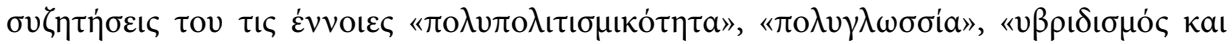

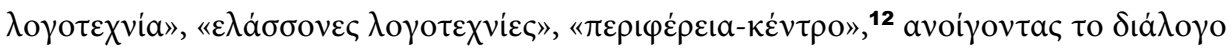

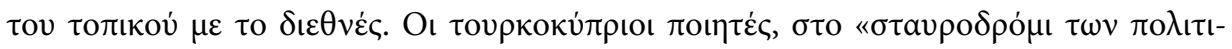

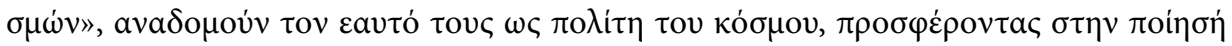

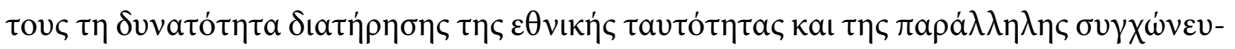

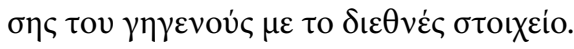

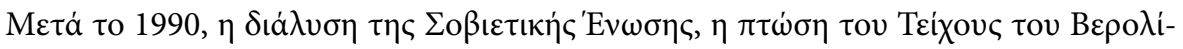

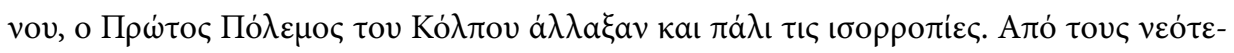

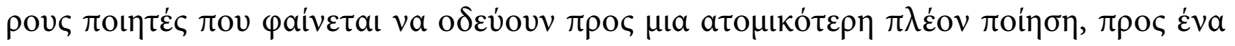

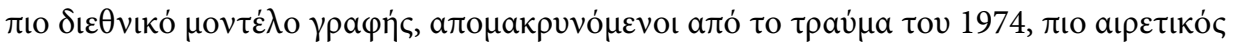

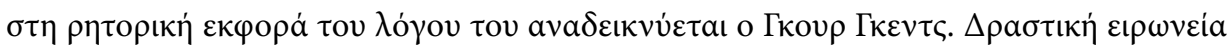
a

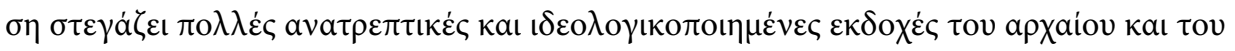

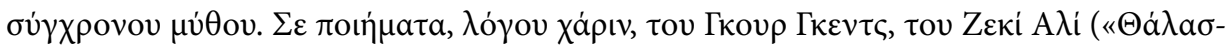

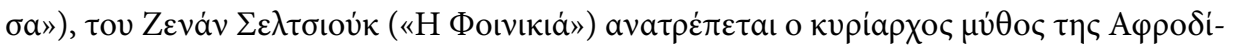

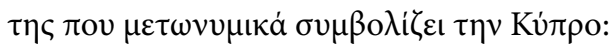

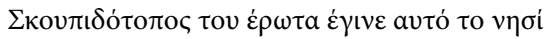

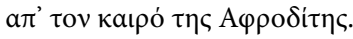

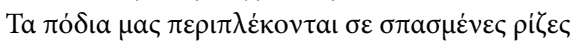

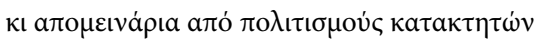

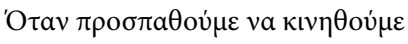

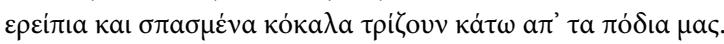

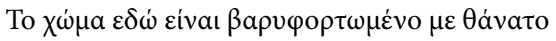

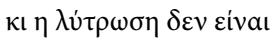

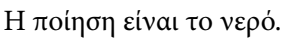

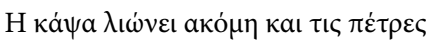

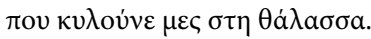

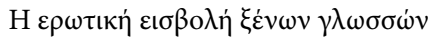

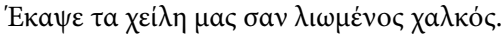

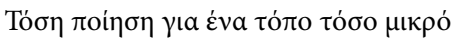

$\pi \alpha \rho \alpha \kappa \alpha \lambda \dot{\omega} \mu \eta \gamma \rho \dot{\varphi} \varphi \varepsilon \tau \varepsilon \dot{\alpha} \lambda \lambda_{0}$

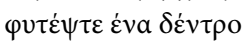

$\varphi v \tau \dot{\varepsilon} \psi \tau \varepsilon v \varepsilon \rho o ́$.

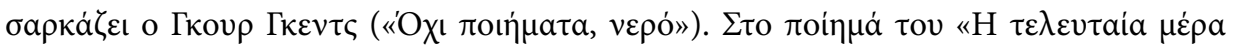

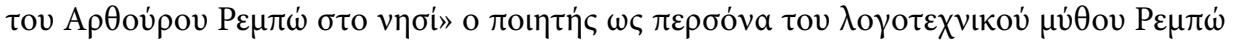

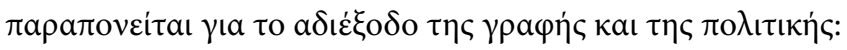




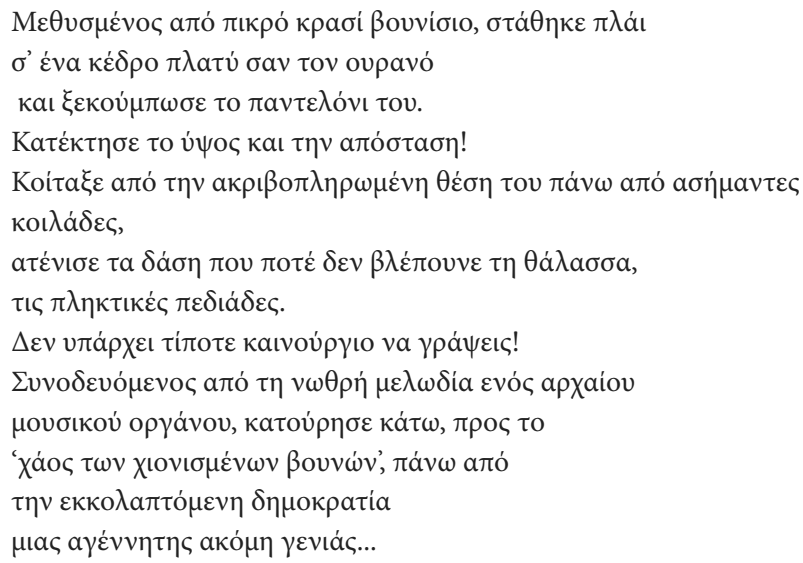

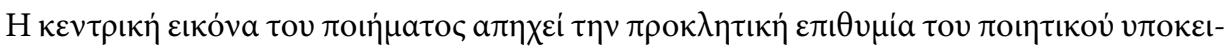

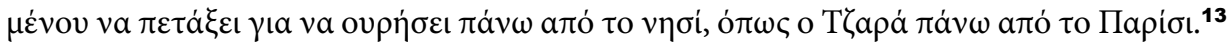

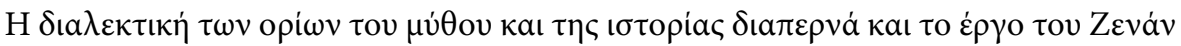

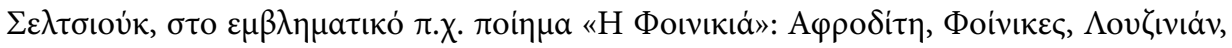

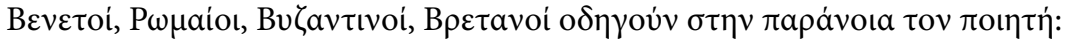

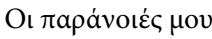

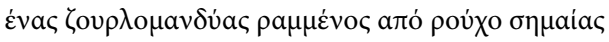

made in Greece, made in Turkey:

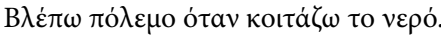

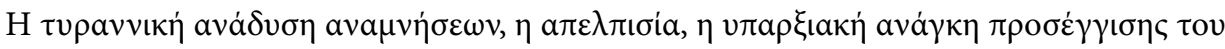

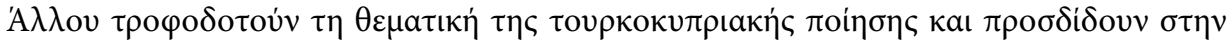

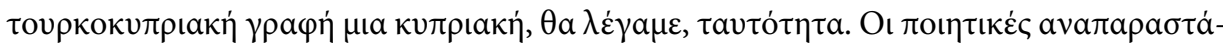

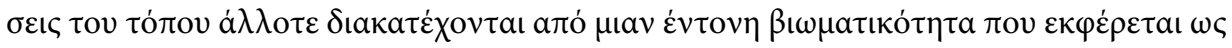

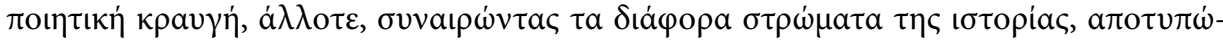

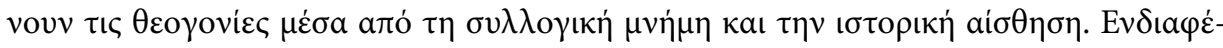

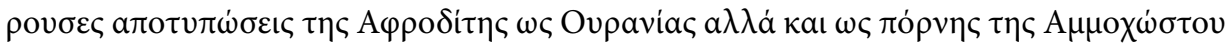

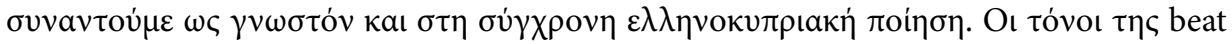

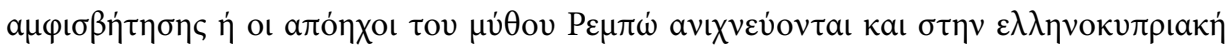

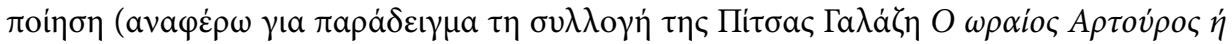

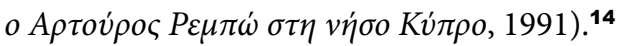

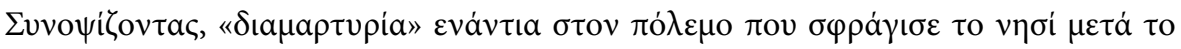

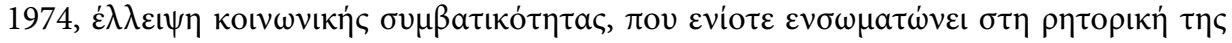

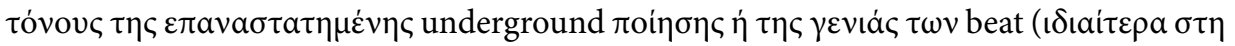

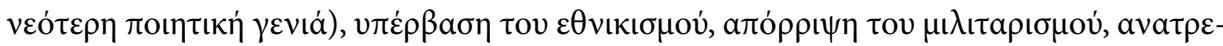

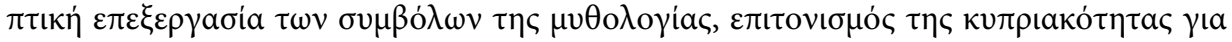




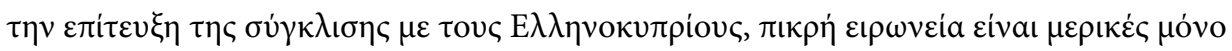
a

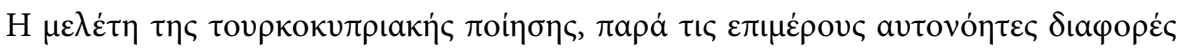

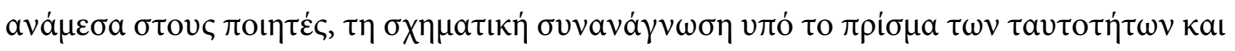

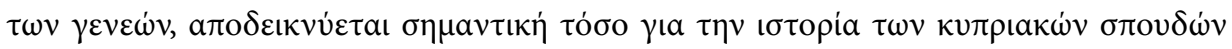

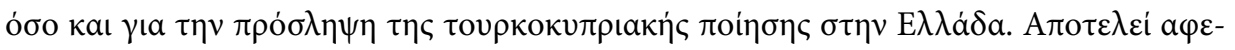

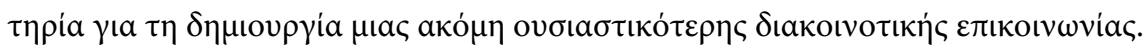

\section{$\Sigma H M E I \Omega \Sigma E I \Sigma$}

$1 \mathrm{~B} \lambda . \pi . \chi$. Keri E. Iyall Smith / Patricia Leavy

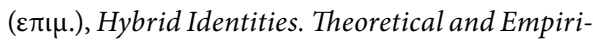

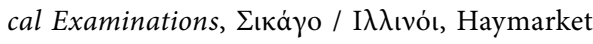

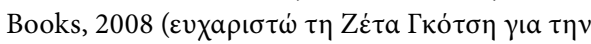

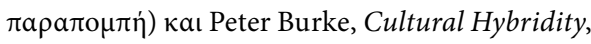

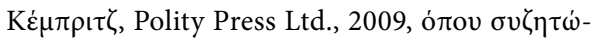

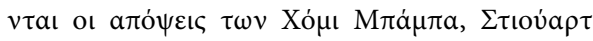

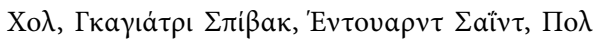

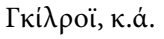

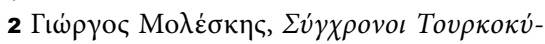

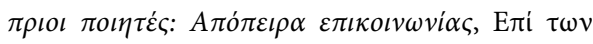

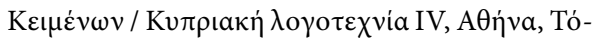

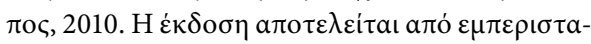

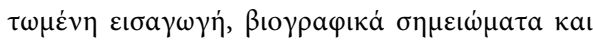

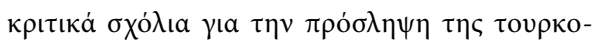

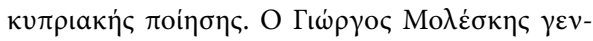

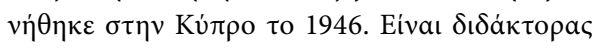

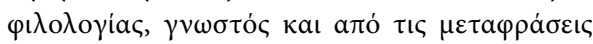

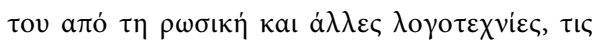

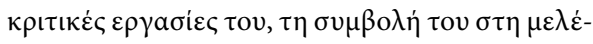

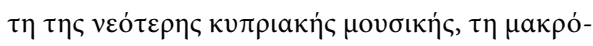

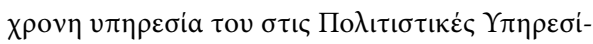

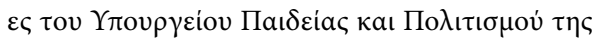

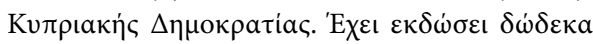

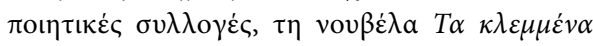

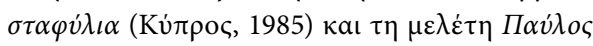

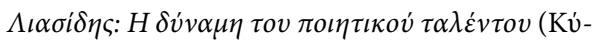

$\pi \rho \circ$, , 1995). Е $\xi \dot{\varepsilon} \delta \omega \sigma \varepsilon, \varepsilon \pi i \sigma \eta \varsigma, \delta v o ~ \sigma v \gamma \kappa \varepsilon v \tau \rho \omega-$

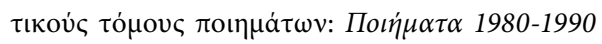

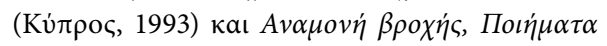

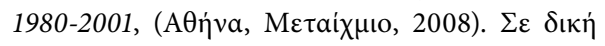

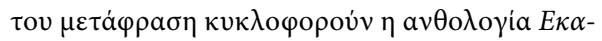

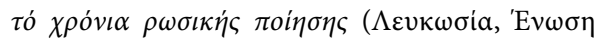

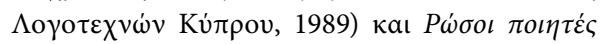

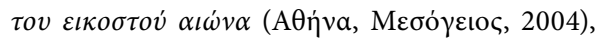

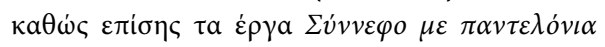

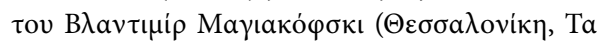

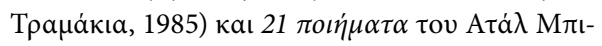

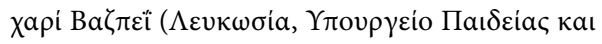

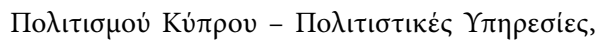

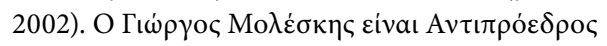

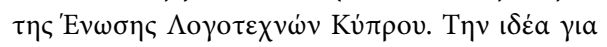

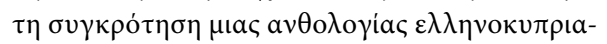

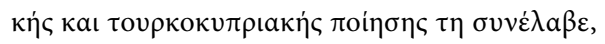

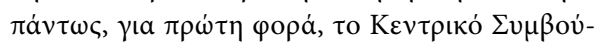

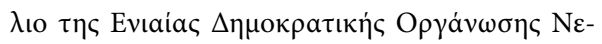

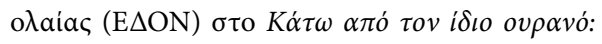

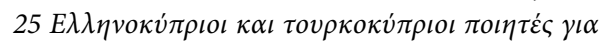

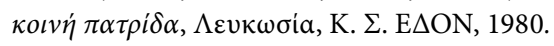

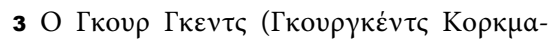

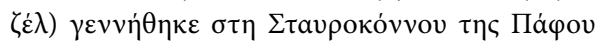

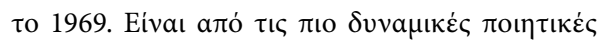

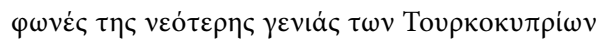

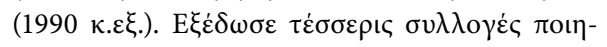




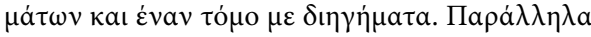

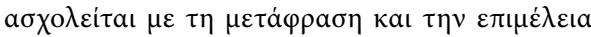
$\varepsilon \kappa \delta$ ó $\varepsilon \omega \nu$. Avá $\mu \varepsilon \sigma \alpha \sigma \varepsilon \dot{\alpha} \lambda \lambda \alpha, \varepsilon \pi \mu \varepsilon \lambda \dot{\eta} \theta \eta \kappa \varepsilon \tau \eta$

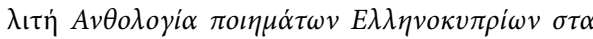

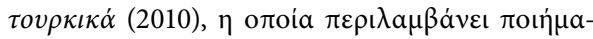

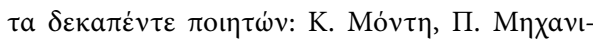

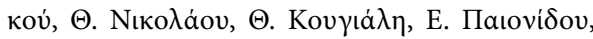

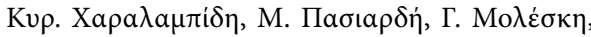

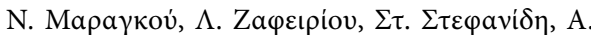

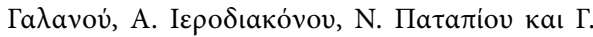
X

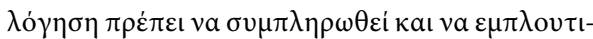

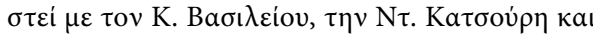

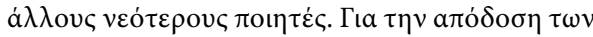

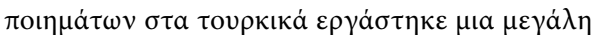

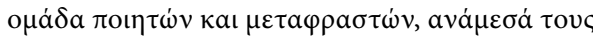

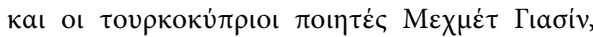

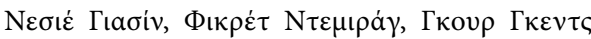

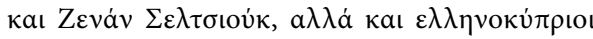

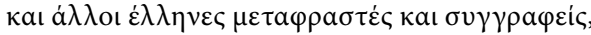

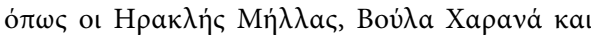

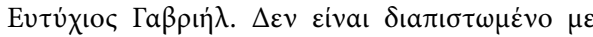

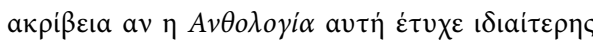

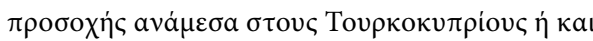

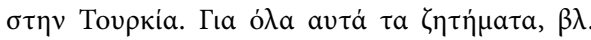

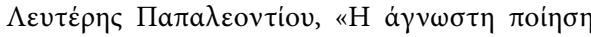

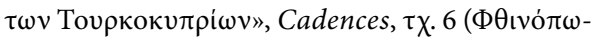
ро 2010), б. 115-117.

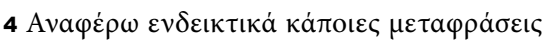

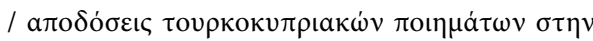

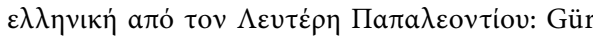

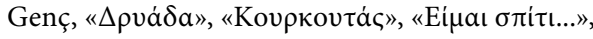

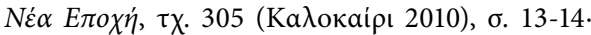

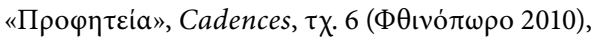

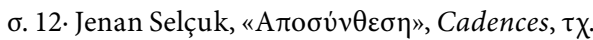

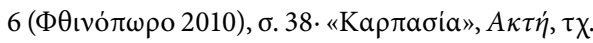

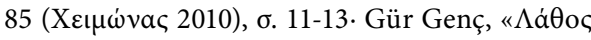

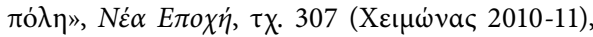

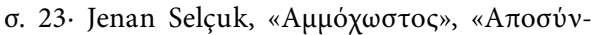

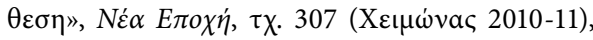

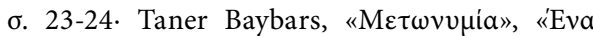

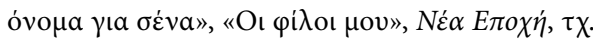

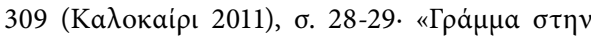

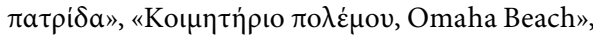

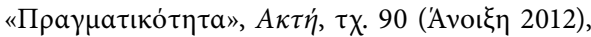

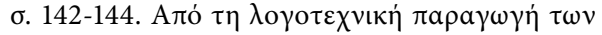

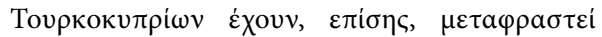

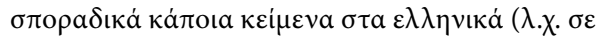

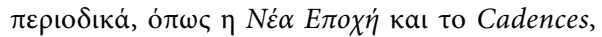

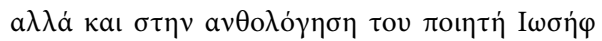

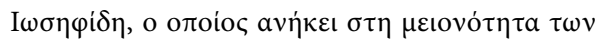

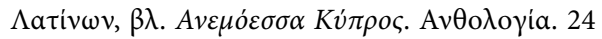

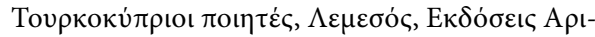

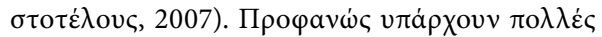

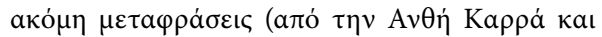

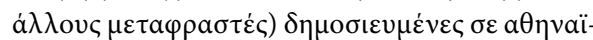

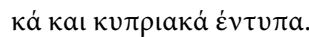

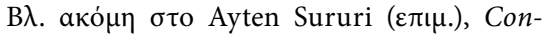

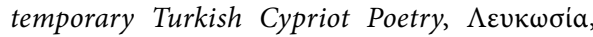
Turkish Cypriot Artist \& Writers' Union, 2008

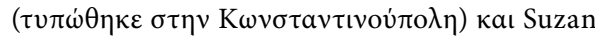

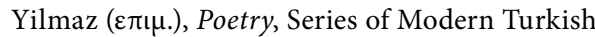

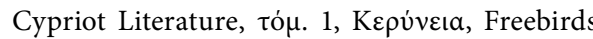

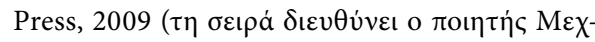
$\mu \dot{\varepsilon} \tau$ Гı $\llcorner\sigma i v)$.

$5 \mathrm{~B} \lambda$. Burke, Cultural Hybridity, $\mu \tau \varphi \rho$. El-

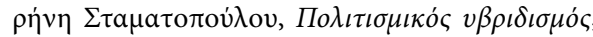

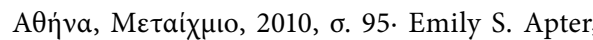
«On Translation in a Global Market», Public

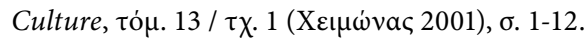

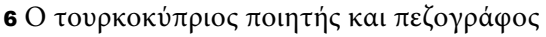

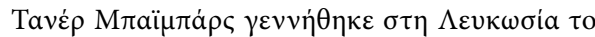

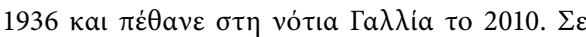

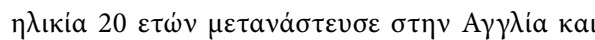

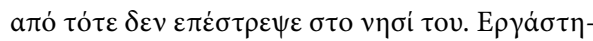

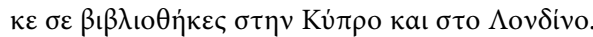

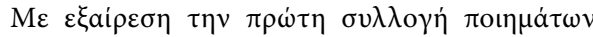

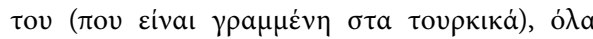

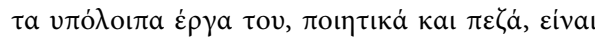

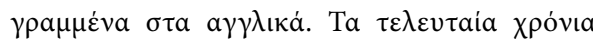

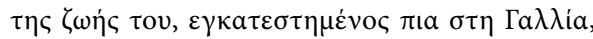

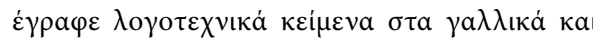

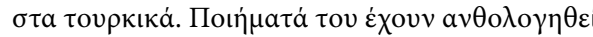

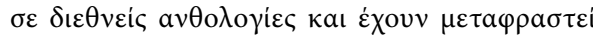

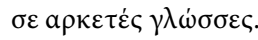

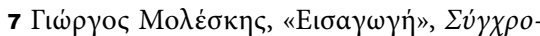

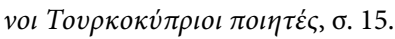

8 В $\lambda . \kappa a ı$ Гเ

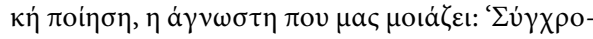

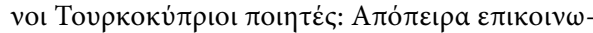




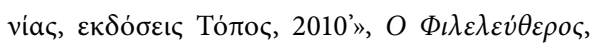
11.12.2011.

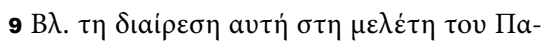

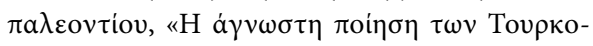

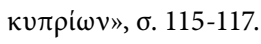

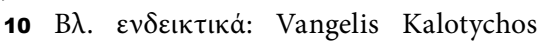

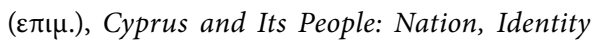
and Experience in an Unimaginable Community

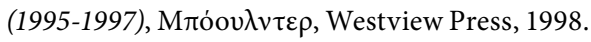

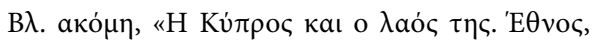

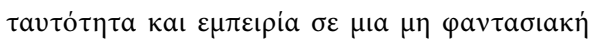

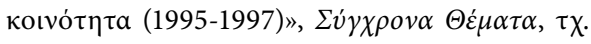

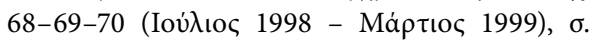
44-64 Matthias Kappler, «Cypriot Literatures as part of the Eastern Mediteranean Contact Area (1850-1960)», Hellenic Studies, 15.2.2007, б. 95-114. П $\beta \lambda$. «Prolegomena for a comparative approach to Cypriot literatures», microphilologica.blogspot.com/2008/01/6-prolegomenafor-comparative-approach.html. A $\lambda \dot{\varepsilon} \xi \eta \varsigma$ Zńpac,

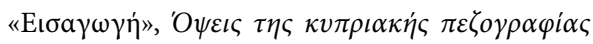

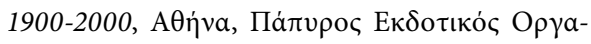

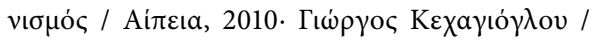

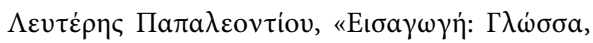

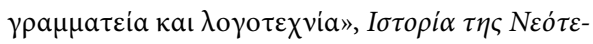

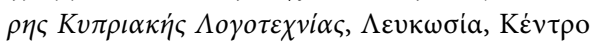

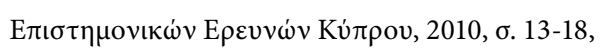

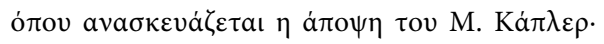

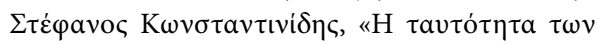

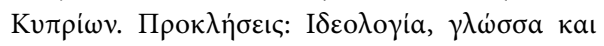

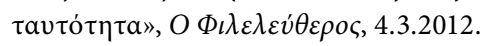

$11 \mathrm{~B} \lambda$. Neşhe Yaşhin, Turkish Cypriot Iden-

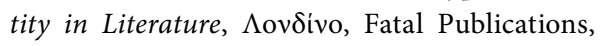

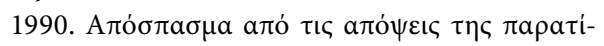

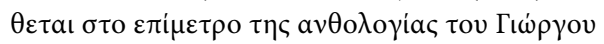

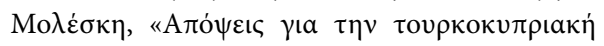

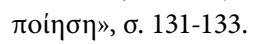

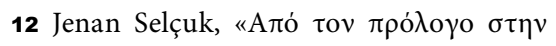

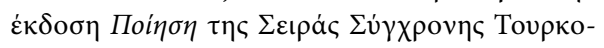

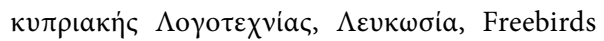

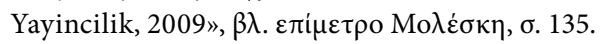

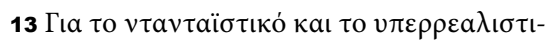
кó $\theta \dot{\varepsilon} \alpha \tau \rho, \beta \lambda$. Henri Béhar, Etude sur le théâtre dada et surréaliste, Парí,, Gallimard, 1967.

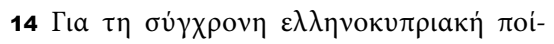

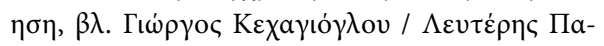

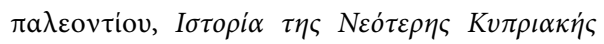

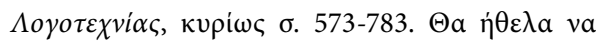

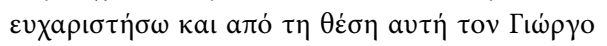

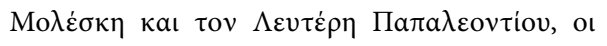

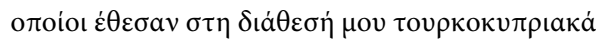

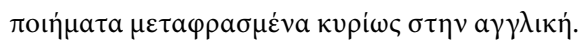

\section{RÉ S U MÉ}

Anna Katsigianni: Lémergence d'une identité culturelle hybride: l'écriture turco-chypriote.

La présente étude explore la stratégie politique de soutien aux frontières culturelles, par le moyen de la poésie. Le dialogue poétique pose les bases des relations intercommunautaires et favorise leur développement. Cet échange de biens culturels entre des poètes et des lecteurs de double culture constitue une «tentative de communication» entre les deux communautés, une forme substantielle de communication à travers la poésie qui contribue à lever les oppositions raciales, nationales, linguistiques, religieuses et historiques. L'osmose culturelle, ce que la critique théorique contemporaine appelle hybridité culturelle, est cultivé de plus en plus systématiquement entre les deux communautés, ce qui ressort des nouvelles publications, extrêmement intéressantes, de poésie gréco-chypriote et turco-chypriote. C’est un événement d'importance littéraire, mais aussi historico-politique que la parution récente de deux importantes anthologies bilingues 
constituées par d'éminents auteurs-traducteurs. La question c'est, en fait, l'élargissement de la multiplicité, de la souplesse et de la nature de l'identité culturelle chypriote. L'identité culturelle «chypriote» des Turco-chypriotes, telle qu'ils la comprennent eux-mêmes principalement, en se différenciant de leur identité turque, est liée à l'élargissement de l'horizon linguistique et culturel de l'écriture chypriote. Pour ce qui est de la question de savoir dans quelle mesure il est possible sur le plan littéraire de regrouper les deux formes d'expression sous le terme générique de «littérature chypriote», dès lors que ce dernier présuppose un sentiment d'identité linguistique, politique et plus largement culturelle, au-delà du fait que l'hétéro-définition est malaisée, la réponse nous est donnée par les Turco-chypriotes eux-mêmes, par leur poésie, qui constitue le prolongement le plus authentique de leur identité sociale (intérêt pour le pays natal blessé, utilisation des symboles mythiques et historiques de l'île, etc.). L'écriture turco-chypriote contemporaine s'épanouit dans un environnement multiculturel et multilinguistique et utilize fréquemment l'outil liguistique de l'Autre, pour diverses raisons - sociales, historiques, politiques, professionnelles, etc. Il serait intéressant de discuter plus largement les points de vue critiques qui ont été formulés sur la question de l'identité et de l'écriture turco-chypriote et les points de vue critiques concernant les différences de réceptivité entre les poètes gréco-chypriotes, dépositaires de la longue tradition littéraire grecque moderne, et turco-chypriotes, dont la poésie a un autre point de départ. 